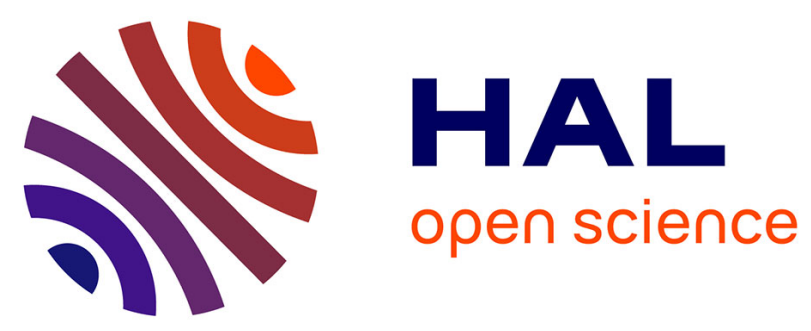

\title{
Experimental Analysis of Multi-hop Routing in Vehicular Ad-hoc Networks
}

Jose Santa, Manabu Tsukada, Thierry Ernst, Antonio F. Gómez-Skarmeta

\section{To cite this version:}

Jose Santa, Manabu Tsukada, Thierry Ernst, Antonio F. Gómez-Skarmeta. Experimental Analysis of Multi-hop Routing in Vehicular Ad-hoc Networks. WEEDEV 2009: 2nd Workshop on Experimental Evaluation and Deployment Experiences on Vehicular networks in conjunction with TRIDENTCOM 2009, Apr 2009, Washington D.C., United States. 10.1109/TRIDENTCOM.2009.4976248 . inria00625645

\section{HAL Id: inria-00625645 https://hal.inria.fr/inria-00625645}

Submitted on 22 Sep 2011

HAL is a multi-disciplinary open access archive for the deposit and dissemination of scientific research documents, whether they are published or not. The documents may come from teaching and research institutions in France or abroad, or from public or private research centers.
L'archive ouverte pluridisciplinaire HAL, est destinée au dépôt et à la diffusion de documents scientifiques de niveau recherche, publiés ou non, émanant des établissements d'enseignement et de recherche français ou étrangers, des laboratoires publics ou privés. 


\title{
Experimental Analysis of Multi-hop Routing in Vehicular Ad-hoc Networks
}

\author{
José Santa*, Manabu Tsukada $^{\dagger}$, Thierry Ernst ${ }^{\dagger}$, Antonio F. Gómez-Skarmeta* \\ *University of Murcia, Department of Information and Communications Engineering \\ Campus de Espinardo, 30100 Murcia, Spain \\ E-mail: \{ josesanta and skarmeta $\} @$ um.es \\ ${ }^{\dagger}$ INRIA Rocquencourt, IMARA Team, Domaine de Voluceau, BP 105, 78153 Le Chesnay Cedex, France \\ E-mail: \{ Manabu.Tsukada and Thierry.Ernst \}@inria.fr
}

\begin{abstract}
Evaluation of vehicular ad-hoc networks (VANETs) over real environments is still a remaining issue for most researchers. There are some works dealing with common 802.11 analysis over real vehicular environments, which carry out performance tests to measure the quality of the communication channel and justify results according to physical and MAC conditions. There are only a few works regarding multi-hop experimentation in this field, and even less (if not none) testing multi-hop protocols. In this paper an integral VANET testbed is evaluated, using 802.11b and a multi-hop network managed by the Optimized Link State Routing protocol (OLSR). Up to four vehicles are used over urban and highway environments to study the VANET performance, and different metrics are used to analyse the results in terms of delay, bandwidth, packet loss and distance between nodes. Furthermore, a deeper analysis is carried out to study the route followed by packets end to end, which enables us to count the number of hops and detect the links where packets are lost. Because a routing protocol is used, results differ from traditional two-hop and staticroute tests, presenting a more realistic study. OLSR is considered as a good reference point for the research community, although it is not the most suitable protocol for vehicular environments, as results show.
\end{abstract}

Index Terms-Vehicular Communications, VANET, Experimental Evaluation, Ad-hoc Networks, Multi-hop communications, OLSR.

\section{INTRODUCTION}

Vehicular networks are becoming essential for telematic services inside the Intelligent Transportation Systems (ITS) field. Apart from autonomous solutions, such entertainment and some collision avoidance systems exclusively based on vehicle sensors, for example, distributed and collaborative services extend the driver perception and integrate the vehicle in the traffic environment. Safety services are the most studied ITS solutions in the current literature, where vehicular networks are commonly used to share navigation and road-side events with the aim of detecting potential hazards. Nonetheless, comfort, traffic management, and monitoring systems, are also more and more dependent on vehicular networks, and implementations of services such as platooning, vehicle tracking, parking reservation and distributed games are only some examples of this expansion.

There are several communication paradigms involved in vehicular networks, which can be essentially summarised as vehicle to vehicle $(\mathrm{V} 2 \mathrm{~V})$, vehicle to infrastructure (V2I), infrastructure to vehicle (I2V), and even infrastructure to infrastructure (I2I). Wireless technologies through 802.11 and cellular networks are the most extended communication links, although their usage depends on concrete applications. Among all possible combinations of communication paradigms and wireless technologies, there is one field specially studied by the ITS community, known as Vehicular Ad-hoc Networks, or VANET. In this case, wireless local area networks, such as 802.11 and DSRC (Dedicated Short Range Communications), are applied into the V2V case using concepts inherited from MANETs (Mobile Ad-hoc Networks).
Although there are a lot of works related to VANET applications and basic research at physical, MAC and, overall, network layers, there is an important lack of real evaluation analysis. Many VANET solutions and protocols could be considered as non practical designs if they were tested over real scenarios, as it has been proved in MANET [1]. Performance of VANET protocols based on a pure broadcast approach can be more or less expected in simple configurations, even if they are not experimentally tested; but the number of issues concerning the real performance of multi-hop designs is much more tricky. As we detail in next section, the amount of works related with real evaluation of VANET designs is limited, rare if we consider the concrete case of multi-hop transmissions, and practically null in the evaluation of routing protocols. Performing real evaluations in VANET research imply a number of issues, most of them inherited from MANET, and even accentuated. Some of the most important drawbacks of performing VANET experiments are:

- Implementation of routing protocols and/or applications.

- Equipment cost.

- Logistic issues in experiments.

- Necessary work to cover a meaningful set of tests.

In this paper, a multi-hop VANET is evaluated over real scenarios, setting-up four cars with the necessary equipment, and involving an interdisciplinary group of people which works on ITS. The goal of the work is becoming a reference point for the VANET community, giving an evaluation of a real multi-hop VANET and IPv6 platform, which uses a standardised ad-hoc routing protocol, as the Optimised Link State Routing (OLSR) protocol is. Testing scenarios have been divided into urban and highway; mobility has been set to static, urban-like speed, and high speed; and a wide range of performance metrics have been used, such as bandwidth, RTT (Round-Trip delay Time), jitter and PDR (Packet Delivery Ratio). Because a low level study has been carried out, it is also possible to count the number of hops and calculate PDR per link in data transmissions. This study is done by a filtering software, which merges information from GPS, dump files and traffic generator traces. Moreover, specific scenarios to test the routing protocol behaviour have been considered. Traffic types used in tests embrace the requirements of most ITS applications, hence ICMPv6, TCP and UDP transmissions have been analysed.

The structure of the paper is organised as follows. In section II, previous works related to VANET experimental evaluation are briefly described. Section III gives a small overview of VANET concepts and OLSR. The experiment set-up, in terms of hardware used, place of tests, considered scenarios, and the software developed to analyse network traffic, is presented in section IV. Results gathered in tests are analysed in section V 
and, finally, the paper is concluded in section VI.

\section{RELATED WORK}

Because of the drawbacks described above, literature in experimental evaluation of VANET architectures is limited, although the presence of such works are of key importance for the ITS community. Up to now, there are several works dealing with this issue, although most of them are still focused on studying the feasibility of 802.11 and DSRC technologies in the vehicular field. In [2] the applicability of $802.11 \mathrm{~b}$ in $\mathrm{V} 2 \mathrm{~V}$ communications is evaluated over urban and highway scenarios, and it is demonstrated that direct line of sight is one of the most important issues of network performance. The hardware platform considered is similar to the one chosen in the current work, based on an embedded PC which acts as access router for in-vehicle devices. A similar analysis is found in [3], where several evaluation metrics are used to quantify the wireless channel performance between two vehicles. Also using 802.11b, in [4] authors demonstrate how mobility and environment factors can seriously degrade the network performance. In addition to consider the most common performance metrics in VANET evaluation, the post-processing software described in next sections, implemented to analyse data logs, enable us to trace packets among communication nodes. Using such feature, it is possible to detect link failures and study the routing protocol performance, in terms of number of hops in transmissions.

Road-side to vehicle communications are also important in ITS, and VANET evaluation papers, as the current one, usually consider this special case in testing scenarios. In [5] the communication link between a static terminal and a moving vehicle is studied in detail. Among all metrics considered, the transmission power is the more original one, determining the maximum communication range. The type of traffic used to test communication performance is also of interest. Most VANET designs use UDP packets, due to poor TCP performance over wireless channels. In [6] and [7] this issue is studied through different configurations; idea also included in current paper.

The previous works only consider two terminals in performance tests, what is not too representative in VANET research. Two recent works evaluate at multi-hop VANET over real scenarios, using three [8] and even six vehicles [9]. These papers offer a wide study about a real VANET set-up, and the last one includes an interesting analysis describing the impact of number of hops on the final performance, what is also treated in the current paper. Nonetheless, static routes are used in that work, presenting a non-realistic vehicular network. Our work, by contrast, considers a real and standardised ad-hoc routing protocol to dynamically modify communication paths. The hardware test-bed presented is also best suited for future ITS research, with a flexible in-vehicle and inter-vehicle IPv6 network based on mobile routers.

\section{VANET CONCEPTS}

The origin of VANET is found in the more general researching subject of ad-hoc networks. However, as it is explained later, vehicular networks present specific conditions which have to be independently studied. OLSR is a well-known proposal of an ad-hoc routing protocol, and its performance in a VANET environment is analysed in this paper.

\section{III-A. Mobile Ad-hoc Network (MANET)}

Mobile Ad-hoc Networks (MANET) are designed to enable wireless communications in dynamic topologies without any infrastructure. In order to adapt to topology changes, MANET nodes exchange control messages to establish the routes used to forward data packets. MANET has the additional advantage of extending the one-hop communication range, since the packets can be delivered through multiple nodes. MANET routing protocols [10] can be classified into the proactive ones, where nodes periodically exchange messages to create routes, and the reactive protocols, in which control messages are exchanged on demand when it is necessary to reach a terminal. Generally, proactive protocols have the advantage of starting communication rapidly by making the routing table ahead, however, this makes battery life shorter due to frequent signalling. If the topology is highly dynamic and the data traffic is frequent, a proactive protocol could be better. Reactive protocols, on the contrary, keeps the battery life longer by reducing signalling messages when there is no data to transmit.

Some routing protocols specified by the IETF MANET working group [11] are: the proactive Optimized Link State Routing (OLSR) and the Topology Dissemination Based on ReversePath Forwarding (TBRPF); and the reactive Ad hoc On-Demand Distance Vector Routing (AODV), the Dynamic Source Routing (DSR) and the Dynamic MANET On-demand (DYMO).

\section{III-B. MANET vs. VANET}

Vehicular Ad-hoc Networks (VANET) [12] are a particular case of MANET, which are characterised by battery constraints free, high speed, GPS-equipped nodes, and regular distribution and movement. First, vehicles have a larger battery than mobile terminals or sensor devices, which is also charged when the engine is started. Second, the speed of vehicles is also higher than common portable terminals, and relative speeds can reach $300 \mathrm{Km} / \mathrm{h}$; hence, the duration of the routing entries is extremely short. Third, a GPS device can be assumed in many cases, whose information improves the network performance in some proposals. Location-Based Multicast (LBM), Greedy Perimeter Stateless Routing (GPSR) and GVGrid, for example, improve routing tasks by using GPS information. Finally, the movement and density of the nodes are not random, since vehicles drive on roads, what makes the nodes position somehow predictive. This concept can be used to detect stable structures or clusters to improve the network performance. Some protocols exploit the locality of vehicles to send packets only to a set of nodes grouped in a geographical zone (geocast), such as GeoGRID.

\section{III-C. OLSR}

OLSR [13] is the routing protocol used in the experiments performed in the work, as it has been stated. Control overhead of this proactive protocol is quite reduced by multipoint relays (MPRs), which characterise OLSR. Each node selects its MPRs among all the one-hop candidates, assuring that all neighbouring nodes at two hops can be reached through a minimum set of them. By using MPRs, the network overhead decreases when node density is high, since only the nodes designated as MPRs forward messages. OLSR nodes detect each other by HELLO messages, which are periodically announced. Topology Control (TC) messages are used to disseminate neighbour information throughout the network. Since OLSR nodes can interconnect different networks, another special message is also periodically disseminated, called Host and Network Association (HNA). In VANETs, this information is necessary, for example, to exchange in-vehicle network addresses. This is the case of the communication platform presented in the paper, where each on-board unit acts as a mobile router (MR) in the vehicular network. 


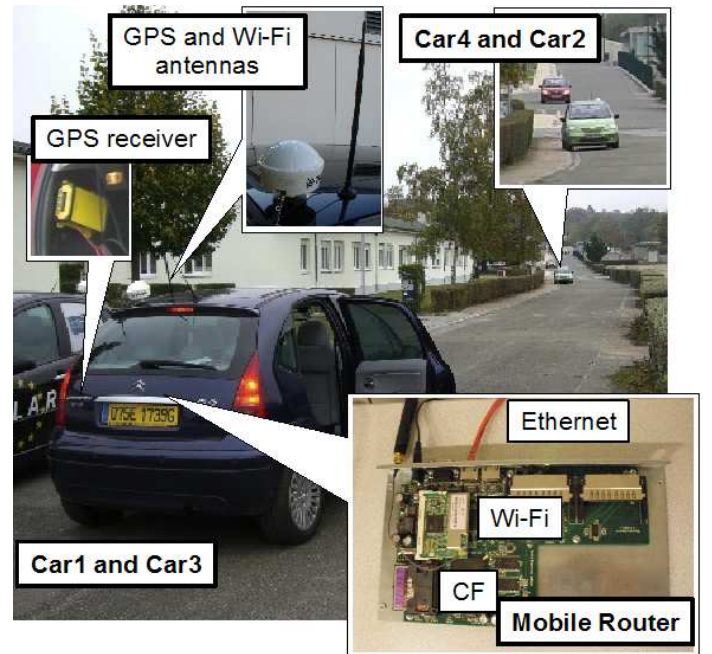

Figure 1. Hardware equipment used in tests

\section{EXPERIMENT SET-UP}

A set of common vehicles have been equipped with the necessary hardware to create a VANET using OLSR. Network traffic and positioning information is logged and then processed in order to analyse the VANET performance. The experiment set-up is described in detail in this section, in terms of hardware and software modules developed, network metrics considered and traffic scenarios.

\section{IV-A. Testbed Platform}

Up to four Citröen C3 cars have been used in the trials, mounting the proper hardware to integrate the vehicle in the VANET and $\log$ positioning and network traffic information. Fig. 1 illustrates the main components of the on-board platform, during one of the field trials. As can be seen, an embedded computer is used as mobile router (MR) in each car. This comprises a Soekris net4521, with a mini-PCI 802.11 Texas Instruments ACX $111802.11 \mathrm{~b} / \mathrm{g}$ wireless transceiver and a compact flash hard disk. The wireless interface has been setup at $11 \mathrm{Mbps}$, emulating an $802.11 \mathrm{~b}$ device. The computer is connected, via serial port, with a Trimble AgGPS 323 GPS receiver, whose external antenna is visible in the photo. The wireless card uses another external antenna, fixed on the car's roof too. One of the two ethernet connections of the MR is used to connect it with the in-vehicle wired network, by means of a hub. In the sender and receiver vehicles, a laptop is connected to the in-vehicle network. The sender laptop is a Windows XPbased system, whereas the second one comprises a Linux Debian computer.

A Linux Voyage distribution with kernel 2.6.22 has been installed on MRs, and the olsr.org daemon 0.5.6-rc7 [14] (an implementation of the OLSR protocol) has been configured on each one. The OLSR configuration parameters are listed in Table I. The transmission period of hello packets has been adjusted to deal with vehicle mobility, through a set of preliminary tests and considering a previous study about tuning OLSR in movility environments [15]. Since the topology is highly variable, the same has been made with TC_Interval and TC_ValidityTime periods. MRs do not use more than one interface in the VANET, hence multiple interface declaration (MID) parameters are left with default values. Host and network association (HNA) parameters have been adjusted, due to the in-vehicle network must be efficiently published to make visible
Table I

OLSR CONFIGURATION PARAMETERS

\begin{tabular}{|c|c|c|}
\hline Parameter & Value & Default \\
\hline Hello_Interval & 0.5 & 2.0 \\
Hello_Validity_Time & 6.0 & 6.0 \\
TC_Interval & 3.0 & 5.0 \\
TC_Validity_Time & 9.0 & 15.0 \\
MID_Interval & 5.0 & 5.0 \\
MID_Validity_Time & 15.0 & 15.0 \\
HNA_Interval & 3.0 & 5.0 \\
HNA_Validity_Time & 9.0 & 15.0 \\
\hline
\end{tabular}

the sender and receiver laptops. The last two parameters should have the same value than the topology control ones [13].

\section{IV-B. Data Gathering and Post-Processing Fusion}

An overview of the experimental evaluation process carried out in the work is given in Fig. 2. In the tests, up to four vehicles have been used, however, the system is prepared to consider any number of vehicles. The sender laptop is in charge of generating data traffic, and both the sender and the receiver ones save a high level $\log$, according to the application used to generate network traffic. All MRs save information about forwarded data packets, by means of the tcpdump software ${ }^{1}$, and log the vehicle position continuously. All this data is analysed in post-process by the AnaVANET software. This is a Java application which traces all the data packets transmitted from the sender node. This way, it is possible to detect packet losses and calculate statistics for each link and end-to-end, and merge all these per-hop information with transport level statistics of the traffic generator. As a result, AnaVANET outputs an XML file with statistics of one-second periods, and a packet trace file with the path followed by each data packet. The first file is uploaded to a Web server, which uses Google Maps functionalities to graphically replay the tests. The Graphic Generator module gives another view of the network performance, using both XML and packet traces to process results and then create several types of figures through the GNU Plot utility.

\section{IV-C. Analysed Traffic and Performance Metrics}

Three different types of data traffic have been considered in the tests carried out over the IPv6 network. These are described in next points, together with the software used to generate the packets:

- UDP. A unidirectional transmission of UDP packets from the sender laptop to the receiver one, has been generated using the IPerf tool ${ }^{2}$. The packet length is 1450 bytes, to avoid IP fragmentation, and they are sent at a rate of 1 Mbps.

- TCP. A TCP connection is established between the sender and receiver laptops, non limiting the maximum bandwidth. The IPerf tool is again used in the traffic generation and the segment size logged in tests was 1440 bytes.

- ICMPv6. The Windows XP Ping6 utility is used to generate IPv6 ICMP (Internet Control Message Protocol) echo request packets from the sender laptop, and to receive echo reply packets from the remote one.

These three types of traffic have been used to analyse the network performance hop-by-hop and end-to-end, considering the most extended metrics in MANET evaluation [10]. In the TCP case, only the high level information given by IPerf, at a 0.5 -second rate, is considered in the process. ICMPv6 and

\footnotetext{
${ }^{1}$ http://www.tcpdump.org/

${ }^{2}$ http://dast.nlanr.net/Projects/Iperf/
} 

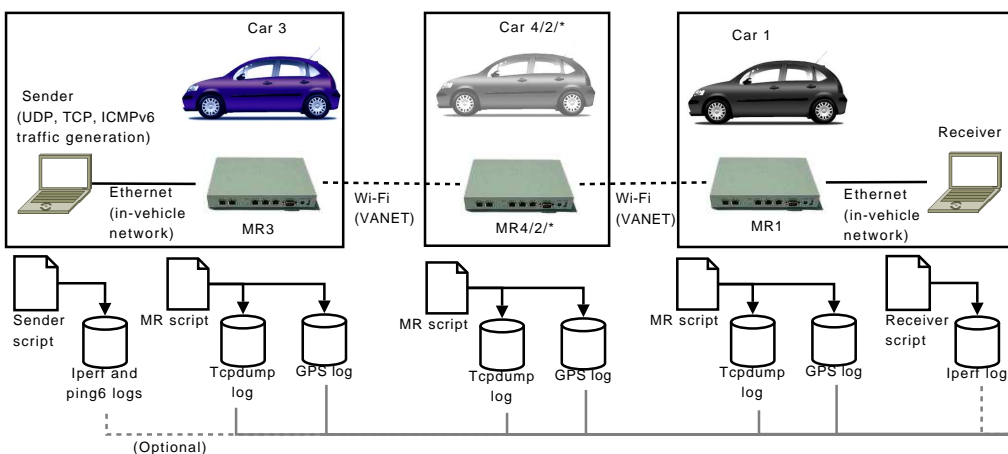

(1)

(Optional)

Figure 2. System overview and data processing units

UDP packets are, however, traced across nodes. Since there is no fragmentation for UDP packets, a direct correspondence exists between MAC and IP layer packets in the study. At this level, the packet delivery ratio (PDR), the number of hops and the jitter are calculated. For ICMPv6 data flows, the round-trip delay time (RTT) have been also considered. At the transport level, the IPerf information used is the instantaneous bandwidth.

\section{IV-D. Experimental Scenarios}

A set of scenarios have been considered with the aim of obtaining significant results under different conditions. The main factors which determine these scenarios are:

- Mobility. Static and dynamic scenarios have been considered to test the network operation under controlled and common traffic settings.

- Environment. Two different environments have been considered: a semi-urban one, inside INRIA-Rocquencourt installations, which contains a set of small buildings surrounded by streets, and a highway stretch, the French A-12, near INRIA-Rocquencourt.

- Number of vehicles. Up to four vehicles are considered in the field trials, in order to check the increase of communication delay with the number of hops.

The set of traffic types (UDP, TCP and ICMPv6) has been applied over each defined scenario. In Fig. 3, four common scenarios in VANET evaluation with up to three vehicles are illustrated. The first one has been used to check the maximum communication range between two vehicles, with the aim of isolating the creation and loss of one link in the OLSR network. The second scenario considers a typical urban environment, where a building (or a set of them) hides the line of sight between the source and the destination cars. A multi-hop network is suited in this kind of situations. A third vehicle forwards the messages received from the source vehicle to the destination one. In the third scenario the three vehicles move around this blockage area. The last scenario in Fig. 3 (number four) uses the same vehicles over a highway environment.

Fig. 4 shows the overtaking and four-vehicle scenarios. The fifth scenario considers static tests with four vehicles, where packets are always forwarded by two intermediate cars. In the sixth one, an overtaking situation is tested, where the black and red vehicles (1 and 2) are parked, and the blue one passes them in the two directions two times. When the distance between the blue and black cars is too big, the direct link is lost, and the OLSR protocol sets-up a multi-hop path using the red car as a relay node. The sender car, after passing the last car in one direction, usually reaches a position where the communication

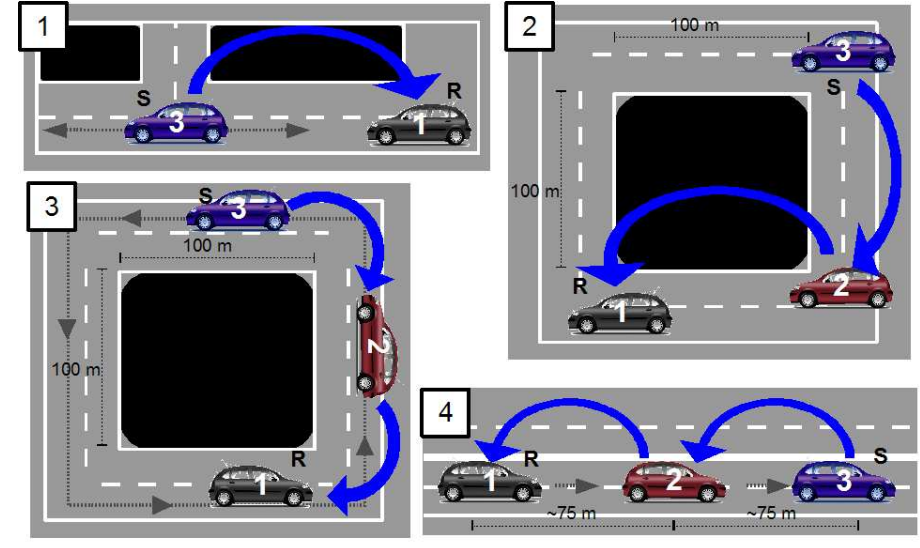

Figure 3. Maximum range and three-vehicles tests
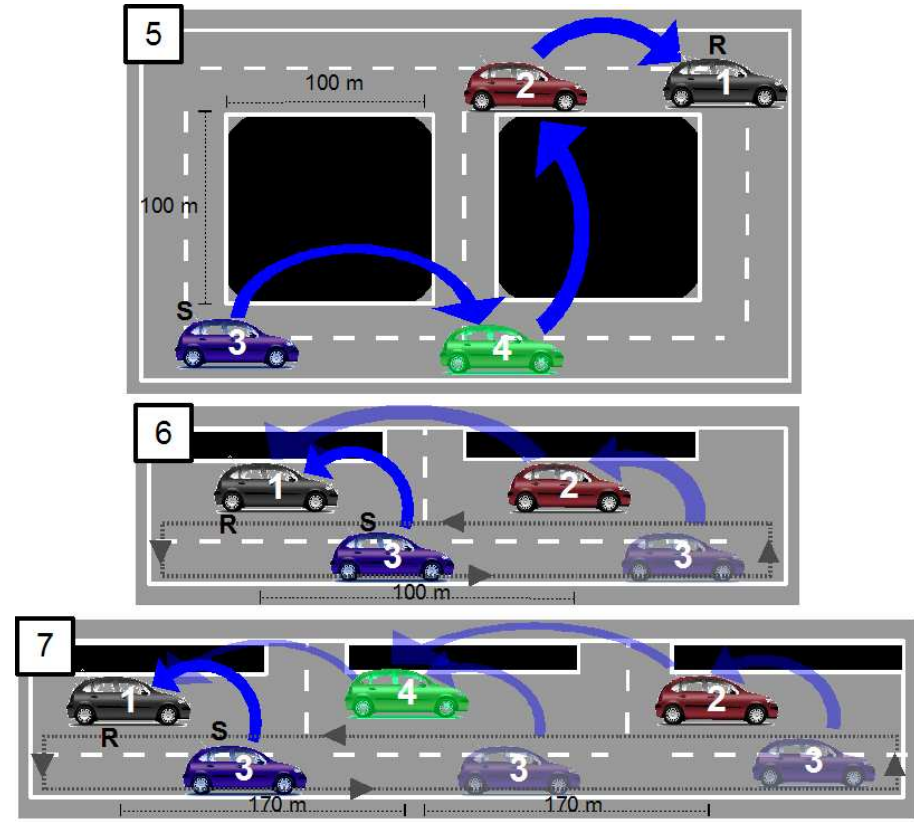

Figure 4. Overtaking and four-vehicles tests

is lost. The last scenario follows the same idea, but four vehicles are now used. The parked vehicles are more separated now, to avoid non-desired direct links.

\section{EVAluation AND ANALYSis}

The VANET experiments described in the previous seven scenarios have been performed at INRIA Rocquencourt, considering the UDP, TCP and ICMPv6 traffic types. Results of the experiments have been processed by AnaVANET and then 


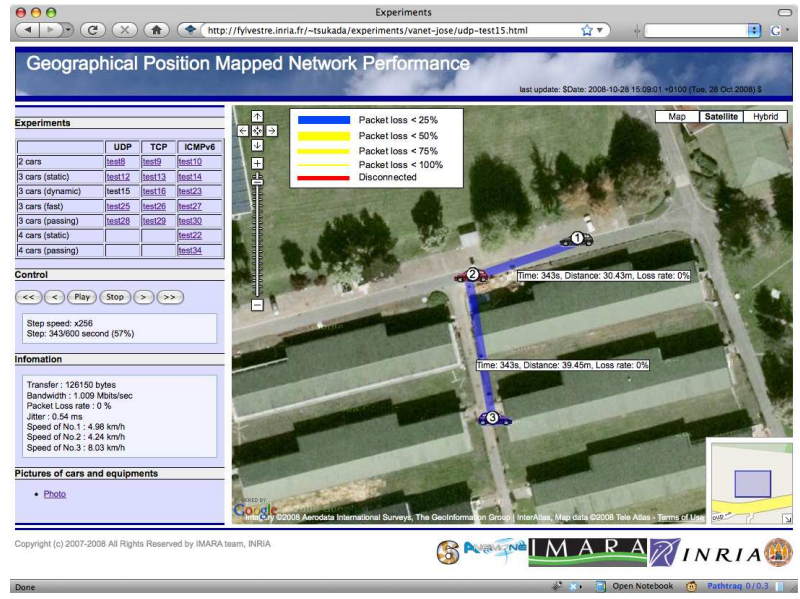

Figure 5. Screenshot of the Report Website.

showed with a graphical software. Moreover, multitude of plots have been generated and used in this section to analyse the main results of the vast experimental evaluation.

\section{V-A. Web-Based Network Analysis}

The carried out experiments are available in our public website $^{3}$, and they can be replayed to see the momentary performance of the network during the tests. Fig. 5 shows a screenshot of the website. All the experiments can be selected and main performance metrics can be monitored at any time. Users can play and stop at any arbitrary point of the test with the control bottons on the left side of the page. The player speed, one step forward and one step backward are also implemented. On the map, the position and movement of the vehicle are depicted with the speed of each vehicle and the distance between them. The transferred data size, bandwidth, packet loss rate, round-trip delay time and jitter, for each link and end to end are displayed. The user can see the network performance by attending the width of link lines and the colour used to draw them.

\section{V-B. Maximum Range Tests}

Maximum range tests have been performed with two cars through Scenario 1. The sender starts leaving from the receiver vehicle position (static), and then it comes back, at about 180 meters, to approach again to the initial point. The speed of the sender was maintained under $10 \mathrm{Km} / \mathrm{h}$ to smoothly check the loss of connectivity.

Fig. 6 shows PDR in the case of the UDP transmission. Packets start to be dropped around 100 meters of distance. The last packet arrives around 120 meters away and, after this point, there are no delivered packets, until the sender vehicle comes back and reaches 100 meters of distance. Since periodical OLSR control messages are lost when the distance is around 120 meters, the path is removed of the routing table and the transmission ends at this point. The jitter in the same test is illustrated in Fig. 7. When the sender car leaves the receiver one, at a distance between 75 and 120 meters, the jitter is higher, due to layer two retransmissions caused by the increase of the distance. When the sender approaches the receiver again, this effect is again visible at distances between 100 and 50 meters. It is noticeable how the communication is lost at a point further away than when the communication comes back. This is due to timeout periods in the reception of control messages give an extra time to maintain the communication link. When the vehicle comes back, some

${ }^{3}$ http://fylvestre.inria.fr/ tsukada/experiments/vanet-jose/
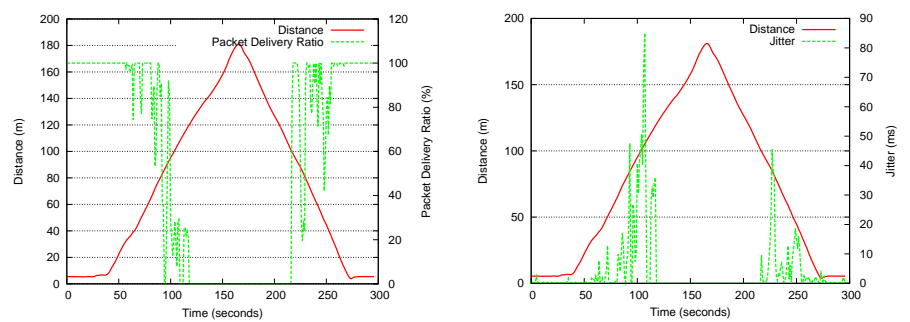

Figure 6. UDP range test with 2 cars Figure 7. UDP range test with 2 cars (distance/PDR)

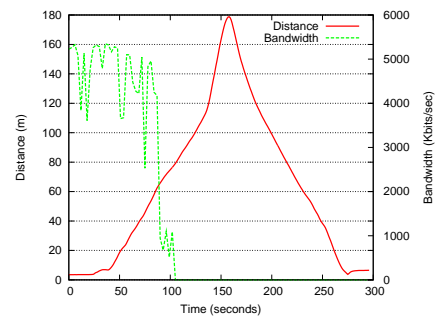
(distance/jitter)

Figure 8. TCP range test with 2 cars Figure 9. Ping range test with 2 cars (distance/bandwidth)

(distance/RTT)

signalling traffic must be also exchanged before the routing table of the sender vehicle is updated.

The TCP performance over the same scenario is showed in Fig. 8. As can be seen, only a one-way path has been logged. When the route is lost at 100 meters of distance, the TCP timeout expires and the transport layer link is broken. Finally, Fig. 9 shows the RTT values collected in a Ping test over the same scenario, measured end to end. The base line of RTT is about $10 \mathrm{~ms}$, but several peaks appear even under good conditions, due to route updates carried out by OLSR and the movement of the sender vehicle. The communication is again lost at a similar distance to the previous cases, however, it comes back earlier than in the UDP test. This is due to the network overhead is much lower in the Ping test (only one message per second), hence the OLSR signalling messages can be efficiently sent and the communication is reestablished earlier.

\section{V-C. Static Tests}

Static results using Scenarios 2 and 5 are summarised in Table II, using three and four vehicles, respectively. The total distance between the sender and receiver cars was 120 meters (70 plus 50 meters) in Scenario 2, and 155 meters (50 plus 70 plus 35 meters) in Scenario 5. As can be seen, the UDP performance is almost ideal. Packet losses are not frequent, and the mean PDR is $99.99 \%$. Small variances of performance are only due to route updates, noticeable in jitter values. In TCP results, the average bandwidth is $1.9 \mathrm{Mbps}$, what reveals a good performance too. However, frequent variations are evident if the standard deviation (STD) is considered. This is due to the operation of the TCP protocol, because the vehicles are static and the network topology does not present variations. According to slow start mechanism, TCP dinamically adjusts the transmission rate, but this algorithm does not converge, due to special features of wireless communications (mainly packet losses) and the presence of eventual route updates.

Ping tests show the good two-way latency of the network. With three vehicles, the average RTT is $4.96 \mathrm{~ms}$, but this value is exceeded when four vehicles are considered, reaching a mean RTT of 7.25. Hence, the addition of one hop increments the latency by more than $2 \mathrm{~ms}$. The RTT standard deviation is also higher in the last case, due to the new node imply additional control traffic and, overall, new occasional route updates. More- 
Table II

NETWORK PERFORMANCE IN STATIC TESTS

\begin{tabular}{|c|l|r|r|r|r|}
\hline Test & Metric & Min. & Ave. & Max. & STD \\
\hline \hline \multirow{2}{*}{ UDP 3 v. } & PDR (\%) & 98.84 & 99.99 & 100 & 0.11 \\
\cline { 2 - 6 } & Bandwidth (Kbps) & 545.20 & 1001.59 & 1020.8 & 34.15 \\
\cline { 2 - 6 } & Jitter (ms) & 0.14 & 0.57 & 5.57 & 0.78 \\
\hline TCP 3 v. & Bandwidth (Kbps) & 327.68 & 1915.95 & 2282.24 & 359.1 \\
\hline Ping 3 v. & RTT (ms) & 4.00 & 4.96 & 23 & 1.38 \\
\hline Ping 4 v. & RTT (ms) & 6.00 & 7.25 & 19 & 1.49 \\
\hline
\end{tabular}

over, since the route from the source to the destination terminals comprises a linear path across the four MRs, as the number of nodes increases, the probability of finding routing or delay problems along the path is higher.

\section{V-D. Dynamic Tests under Urban Conditions}

According to Scenario 3, three vehicles have been driven around a set of buildings, with the intention of blocking the direct link between Cars 3 and 1. The speed of the test where set between $15 \mathrm{~km} / \mathrm{h}$ and $30 \mathrm{~km} / \mathrm{h}$. The right and left roads illustrated in Scenario 3 are in reality very narrow, hence some communications problems appear in the corners.

The results collected in the UDP test are plotted in Fig. 10. The upper plot shows the number of hops used in the paths followed by UDP packets, whereas the lower graphs show the PDR, computed end to end and per link. PDR is calculated per second, while the number of hops is plotted for each packet transmitted from the sender node. When no hops are drawn, the route to the destination vehicle is not available. Zero hops means that the packet was sent by the first MR, but it was not received by any other. Negative values represent those packets which did not arrive to the destination vehicle, but some hops were reached. As can be seen, a direct relation exists between PDR and number of hops. When this last value is equal or lower than zero, the PDR decreases. When the vehicles are in the same street, some direct paths (one-hop) appear; however, when the distance between the sender and the receiver cars is large enough, the two-hop route is used. These different types of paths can be also seen if the perlink PDR is observed. Whereas the direct link (MR3-MR1) gives intermediate PDR values, the PDR between consecutive vehicles is almost almost identical and near $100 \%$ when the two-hop link is used, due to the lower distance between nodes.

Since communication problems appear at corners in some laps, OLSR signalling messages are lost, and the communication is temporary down. An interesting effect is also noticeable at time 250 seconds of the UDP test. Here, several paths with more than two hops are registered. This is due to routing problems of OLSR when the destination node suddenly disappear, what provokes cycles in the network. When the network topology is finally updated in all the nodes, these packets are finally dropped, if the receiver node is out of range, or they reach the destination. These cycles provoke a great eventual jitter, which is also highly variable in the rest of the test, due to stressful conditions.

The bandwidth obtained in the TCP test is showed in Fig. 11. The performance of the network is very good in the first fifty seconds, due to the vehicles started the trial parked very near. However, the rest of the test shows a high variability, due to continuous changes in topology and communication problems in corners. When conditions are favourable, TCP try to normalise the bandwith, but soon a link disappears and the bandwidth falls. Peaks of performance are obtained when the sender and receiver cars are in a direct line of sight. TCP timeouts do not expire because there are no long disconnection periods, hence the transport-level communication is maintained.

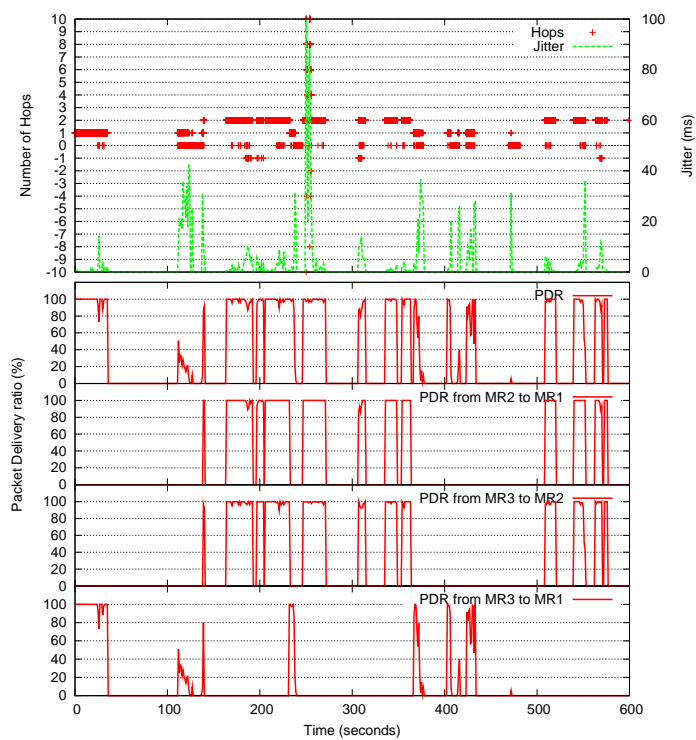

Figure 10. UDP urban test with 3 dynamic cars
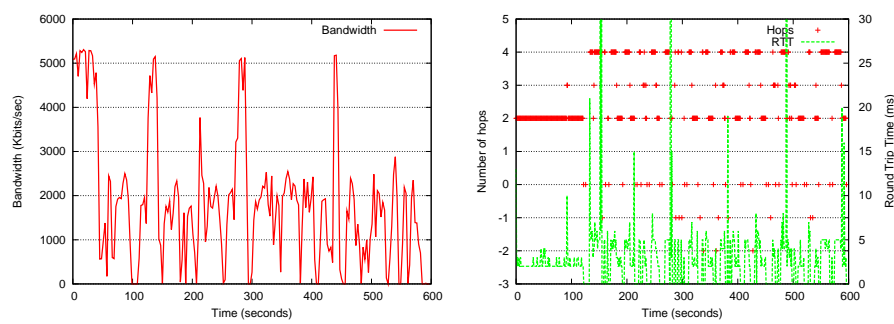

Figure 11. TCP urban test with 3 Figure 12. Ping urban test with 3 dynamic cars (bandwidth) dynamic cars (hops/RTT)

The final test (Fig. 12) comprises a Ping transmission. As can be seen, several steps appear between two main RTT values: five and seven milliseconds. This match with two-hop and four-hop two-way paths. Several three-hop routes have been collected, due to, sometimes, the ICMP Echo Request packets take a different route than the Echo Reply ones. If the ratio of non delivered packets (negative hop counts in this case) is compared with the one obtained in the UDP test, it is noticeable how it is lower now. Since the data traffic is much more lower in the Ping case (one message per second), signalling traffic is more efficiently propagated, and changes in network topology are earlier known.

\section{V-E. Dynamic Tests in Highway}

The dynamic tests performed over highway conditions follow Scenario 4 . The speed of the cars was around $100 \mathrm{~km} / \mathrm{h}$, but the distance between vehicles was variable, due to the rest of traffic on the road. Moreover, communication problems in this test are not only due to buildings, but also to surrounding vehicles.

The PDR obtained in the UDP test is presented in the lower part of Fig. 13. As can be seen, when the distance between vehicles increases, the PDR becomes lower. As in the urban scenario, intermediate values between 0 and $100 \%$ are not very frequent, due to OLSR remove the routes between nodes when signaling packets are lost. At the beginning, the network performance is good, due to the direct path is chosen, as can be seen in the partial PDR study of the MR3-MR1 link. When vehicles start to separate, the two-hop path is used, as it is showed in the PDR of MR2-MR1 and MR3-MR2 links and the number of hops of chosen paths, showed in the upper graph. High variations of distance provoke route updates and, therefore, packet losses. Around 300 seconds of test, vehicles regroup, but 


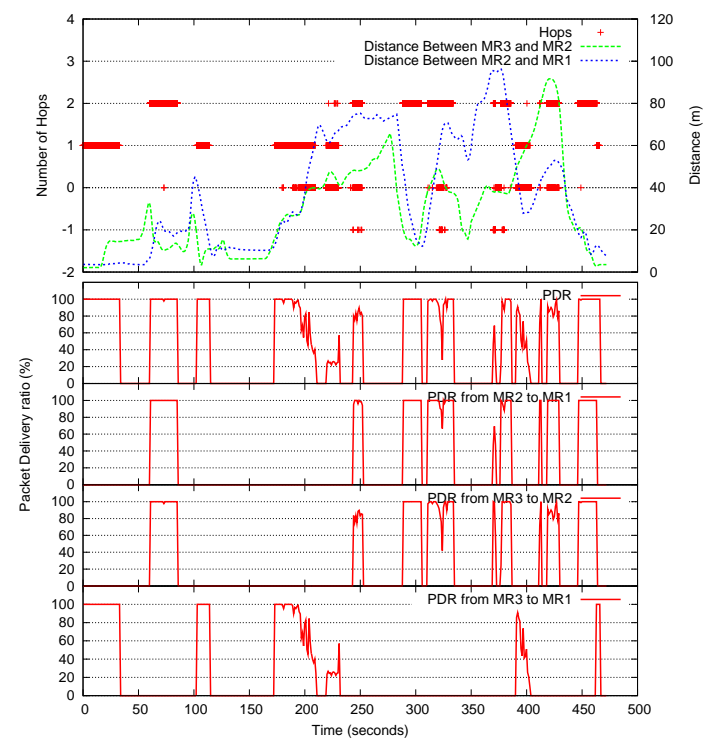

Figure 13. UDP highway test with 3 cars

the three-hop path is maintained, due to the time OLSR needs to adapt to the new topology. The high variability of distance around time 350 seconds, makes the network does not stabilise and many packets are lost. A higher period of $0 \%$ of PDR is noticeable, however, around 150 seconds of test. In this case, the communication between the sender vehicle and the others is blocked by a near building.

The bandwidth results of the TCP test are showed in Fig. 14. Now the vehicles are grouped at the beginning of the tests and the bandwidth is around $5 \mathrm{Mbps}$. However, when Car 3 enters the highway and the distance with the other two cars increase, the bandwidth dramatically falls. As can be seen during the whole test, there is again a direct relation between the distance of vehicles and the final performance. Taking into account the maximum range and static tests, it is easy to identify in the graph the moments in which a three-hop path is used. Bandwidths around 2 Mbps represent these cases, whereas results between 4 and $5 \mathrm{Mbps}$ belong to direct paths.

Finally, Fig. 15-16 show the results collected during the Ping test. As can be seen, the RTT increases when the vehicles are far enough to use a four-hop two-way route. At this moment, the RTT passes from around three milliseconds to reach the five milliseconds. It is advisable again, how intermediate RTT values are not frequent, being the number of hops the main factor which determines the result. When the distance among vehicles grows and communication starts to be difficult, the links between MRs break, due to losses of OLSR signalling messages.

\section{V-F. Overtaking Tests}

The overtaking tests using three and four vehicles have been carried out according to Scenarios 6 and 7. Two and three cars, respectively, were parked at a straight avenue, to simulate they are driving at the same speed, and the sender vehicle overtakes them several times in both directions (see Fig. 4).

The results of the UDP test with three vehicles are depicted in Fig. 17. The distances are calculated taking into account the moving vehicle (Car 3) as the reference. It can be seen that twohop routes appear when the sender vehicle is around the non receiver one. However, during periods of route updates, packets are not delivered. Since the parked vehicles are in an open area and they have a direct line of sight, there are no practically packet losses in the MR1-MR2 link. It is advisable how the number of correctly delivered packets is greater between peaks of distance, but shifted to the left. This is due to the time required by OLSR to adapt to the new topology. Therefore, the best results are obtained when the routes are maintained for a long time. This is the case when the sender car comes back after "overtaking" the other two, since the appropriate route was established when the sender vehicle passed them. Fig. 17 also reflects that a high jitter is maintained during all the test, due to high dynamism of the network. At the beginning, jitter starts to increase when the sender vehicle goes away of its initial position and, during the rest of the test, it is highly variable due to route updates.

The bandwidth results of the TCP test are plotted in Fig. 18. As in the previous TCP tests, the two characteristic values around 5 and 2 Mbps are again visible when one-hop and two-hop routes are established, respectively. As can be seen, the direct path is used when the distance between the sender and receiver cars is lower. The distance curves are now a bit different, because the sender vehicle comes back earlier after passing both cars, in order to maintain the TCP session.

Two ping tests with three and four vehicles were made following the same overtaking pattern. Fig. 19 shows the results of the first one. RTT fluctuations between three and five milliseconds, depend wether the two or four-hop two-way paths are chosen. Some three-hop paths have been collected, due to the different path of some Echo Request and Echo Reply messages. As can be seen, the behaviour of the network is more regular than in the UDP case, and packet losses are mostly due to peaks of distance. This is explained again by the low data traffic, which allows OLSR control messages to be efficiently transmitted. This way, route updates due to topology changes are made faster. In the four-vehicle test of Fig. 20, the paths are more varied. However, one-way paths of three hops are not frequent. OLSR bet on maintaining two-hop paths when communication is possible, hence Car 2 is bypassed when it is "overtaken" the first time. In the second passing of this car, some two-way paths of six hops are collected, however. More packet losses have been collected than in the previous Ping test, because the parked vehicles were further separated to avoid direct paths among them

\section{CONCLUSIONS}

The work presented in this paper offers a complete testbed for VANET evaluation, suited to carry out performance trials over the IPv6 basis, but, overall, it gives an original evaluation of an ad-hoc routing protocol in the vehicle domain. The platform enables the researcher to analyse the network performance at low level. Several logs collected from individual mobile routers are post-processed to calculate several performance metrics at link, network and transport level. This way, typical statistics are obtained, such as the packet delivery ratio, round-trip delay time, jitter and bandwidth; but also new performance metrics are offered, such as the number of hops used to deliver a packet, or the per-link PDR, for example.

Up to four vehicles have been set-up to perform multitude of tests in the surroundings of INRIA Ronquencourt. These cover both urban and highway environments, and take into account static and dynamic conditions. Main results of all these field trials have been analysed in the paper. The results show the great performance difference which can be found if a routing protocol is used in VANET evaluation, in comparison to previous experimental analysis available in the literature based on static routes. Although it has been tuned to dynamic conditions, the OLSR protocol shows limitations to efficiently update routing tables under stressful conditions. This effect is more noticeable when 


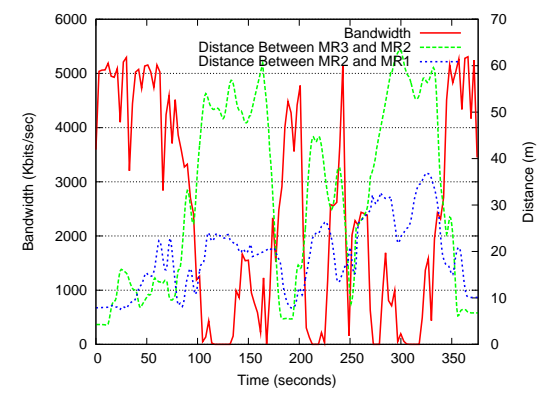

Figure 14. TCP highway test with 3 dynamic cars (bandwidth/distance)

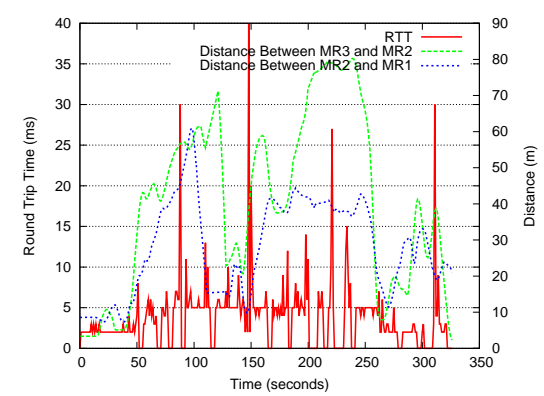

Figure 15. Ping highway test with 3 dynamic Figure 16. Ping highway test with 3 dynamic cars (RTT/distance)

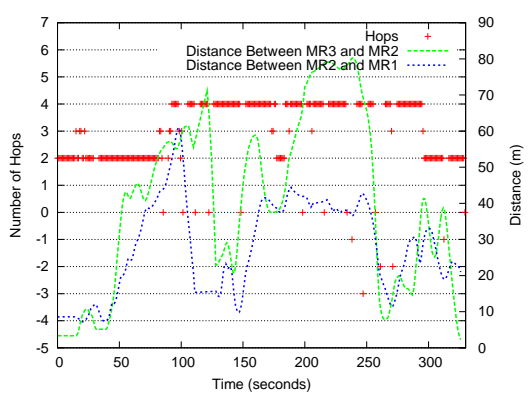

cars (hops/distance)

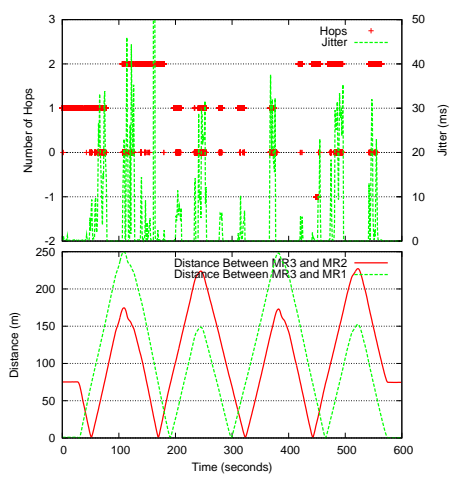

Figure 17. UDP overtaking test with 3 Figur

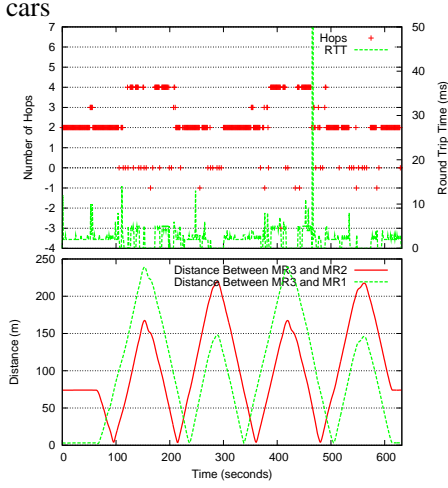

Figure 19. Ping overtaking test with 3 Figure 20. Ping overtaking test with 4 cars cars

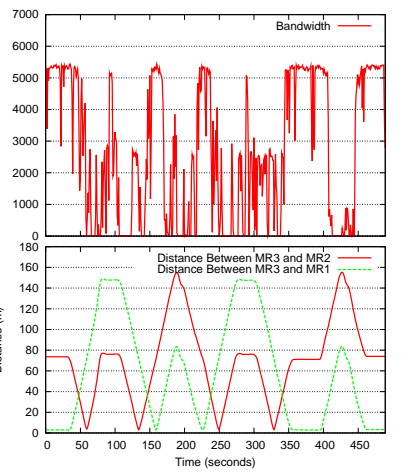

cars

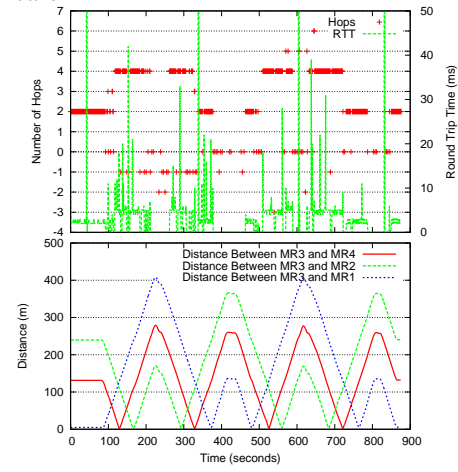

the volume of data traffic is high, due to network overload. The maximum communication range between vehicles also presents a different pattern, if we compare the results with the ones obtained in static route configurations. Now, the communication is cut when some control messages are lost and OLSR timeouts expire. Hence, low PDR are less frequent than in static route tests.

The TCP operation over real VANET deployments should be specially taken into account, because the lack of routing information for a while can lead to transport-level disconnection. In all the tests, the line of sight between vehicles has been a key factor to maintain communication links. Moreover, the number of hops used in transmission paths, has been identified as another key performance factor. An incremental delay between two and three milliseconds per hop has been detected when direct paths between nodes are not used. These cases can be found when the distance between sender and receiver vehicles increase significantly, or when near buildings block the direct communication. However, it has been checked that OLSR prefers smaller paths when communication is possible.

In the future, the data recorded during the vast set of tests will be further exploited. A VANET-oriented protocol developed at INRIA will be also evaluated through new field trials, using the presented test-bed. This is located inside the geographic-based routing proposals, which are demonstrating to be suitable for the road domain. At the University of Murcia, ongoing cellular network evaluations will be soon complemented with WiMAX trials in the vehicular frame.

\section{ACKNOWLEDGEMENTS}

This work has been carried out in frames of the Spanish Programa de Ayuda a los Grupos de Excelencia de la Fundación Séneca 04552/GERM/06, the CICYT project TIN2008-06441C02-02 and the FPU grant AP2005-1437 from the Spanish ${ }_{3}$ Ministerio de Ciencia e Innovación.

\section{REFERENCES}

[1] C. Tschudin, H. Lundgren, and E. Nordstrom, "Embedding MANETs in the real world," Lecture notes in computer science, vol. 2775, no. 1, pp. 578-589, September 2003.

[2] V. González, A. L. Santos, C. Pinart, and F. Milagro, "Experimental demonstration of the viability of IEEE $802.11 \mathrm{~b}$ based inter-vehicle communications," in TridentCom '08: Proceedings of the 4th International Conference on Testbeds and research infrastructures for the development of networks \& communities, 2008, pp. 1-7.

[3] M. Jerbi, P. Marlier, and S. M. Senouci, "Experimental assessment of $\mathrm{V} 2 \mathrm{~V}$ and I2V communications," in Proc. IEEE Internatonal Conference on Mobile Adhoc and Sensor Systems MASS 2007, 2007, pp. 1-6.

[4] J. Singh, N. Bambos, B. Srinivasan, and D. Clawin, "Wireless LAN performance under varied stress conditions in vehicular traffic scenarios," in Vehicular Technology Conference Fall, vol. 2, Vancouver, Canada, September 2002, pp. 743-747.

[5] C. Wewetzer, M. Caliskan, K. Meier, and A. Luebke, "Experimental evaluation of UMTS and wireless LAN for inter-vehicle communication," in International Conference ITS Telecommunications, Sophia Antipolis, France, June 2007, pp. 287-292.

[6] F. Hui and P. Mohapatra, "Experimental characterization of multi-hop communications in vehicular ad hoc network," in ACM international workshop on Vehicular ad hoc networks, Cologne, Germany, September 2005, pp. 85-86.

[7] A. Festag, H. Fubler, H. Hartenstein, A. Sarma, and R. Schmitz, "Fleetnet: Bringing car-to-car communication into the real world," in ITS World Congress, Nagoya, Japan, October 2004.

[8] M. Jerbi, S. M. Senouci, and M. Al Haj, "Extensive experimental characterization of communications in vehicular ad hoc networks within different environments," in Proc. VTC2007-Spring Vehicular Technology Conference IEEE 65th, 2007, pp. 2590-2594.

[9] M. Jerbi and S. M. Senouci, "Characterizing multi-hop communication in vehicular networks," in Proc. IEEE Wireless Communications and Networking Conference WCNC 2008, 2008, pp. 3309-3313.

[10] Z. Chang, G. Gaydadjiev, and S. Vassiliadis, "Routing protocols for mobile ad-hoc networks: Current development and evaluations," in Annual Workshop on Circuits, Systems and Signal Processing, Veldhoven, Holland, November 2005, pp. 489-494.

[11] "IETF: Mobile Ad-hoc Networks (MANET) Working Group," 2008, http://www.ietf.org/html.charters/manet-charter.html.

[12] F. Li and Y. Wang, "Routing in vehicular ad hoc networks: A survey," IEEE Vehicular Technology Magazine, vol. 2, no. 2, pp. 12-22, June 2007.

[13] T. Clausen and P. Jaquet, Optimized Link State Routing protocol (OLSR), IETF MANET Working Group, October 2003, RFC 3626.

[14] o. O. daemon, "http://http://www.olsr.org/."

[15] Y. Cheng, S. Bhatti, and D. Parker, "Tuning olss," in IEEE International Symposium on Personal, Indoor and Mobile Radio Communications, Helsinki, Finland, September 2006, pp. 1-5. 